EPJ Web of Conferences 52, 06006 (2013)

DOI: 10.1051/epjconf/20135206006

(C) Owned by the authors, published by EDP Sciences, 2013

\title{
CRPropa 2.0
}

\section{Public Software to Model Extragalactic Propagation of Ultra-High Energy Nuclei}

\author{
Arjen van Vliet ${ }^{1}$, Karl-Heinz Kampert ${ }^{2}$, Jörg Kulbartz ${ }^{1}$, Luca Maccione ${ }^{3,4}$, Nils Nierstenhoefer ${ }^{1,2}$, Peter Schiffer $^{1}$, and \\ Günter Sigl ${ }^{1}$ \\ ${ }^{1}$ II. Institut für Theoretische Physik, Universität Hamburg, Luruper Chaussee 149, 22761 Hamburg, Germany \\ ${ }^{2}$ University of Wuppertal, Gaußstraße 20, 42119 Wuppertal, Germany \\ ${ }^{3}$ Ludwig-Maximilians-Universität, Arnold Sommerfeld Center, Theresienstraße 37, D-80333 München \\ ${ }^{4}$ Max-Planck-Institut für Physik (Werner-Heisenberg-Institut), Föhringer Ring 6, D-80805 München
}

\begin{abstract}
Recently CRPropa 2.0 has been released as a public software package to simulate the extragalactic propagation of ultra-high energy nuclei. It includes deflections by extragalactic magnetic fields and all relevant interactions with ambient photon backgrounds. Furthermore it provides the possibility to track secondary $\gamma$-rays and neutrinos produced in the different reactions. This allows for the investigation of their connection with the primary nuclei, the so called multi-messenger approach. In this paper CRPropa 2.0 is introduced, its different components are discussed and two example applications are presented.
\end{abstract}

\section{Introduction}

Ultra-high energy cosmic rays (UHECRs), ionized nuclei with energies that exceed $10^{18} \mathrm{eV}$, have been a subject of intense research for more than 70 years. However their sources, mass and propagation mechanisms are all still up for debate. Arrival-direction data of the Pierre Auger Observatory indicate a correlation with the position in the sky of nearby active galactic nuclei (AGNs) or more generally with the nearby extragalactic matter distribution [1]. Mass composition measurements by the Pierre Auger Collaboration suggest a significant fraction of heavy primaries above $10^{19} \mathrm{eV}$ [2], whereas data from HiRes and from the Telescope Array [3, 4] suggest a proton dominance in the same energy range. The flux-suppression in the energy spectrum above about $5 \cdot 10^{19} \mathrm{eV}$, as has been observed by HiRes, the Pierre Auger Observatory and the Telescope Array [4-6], could be a consequence of the GZK-effect $[7,8]$, or of an energy limit in the acceleration at the sources. To resolve these issues, a better understanding of the effects of cosmic ray propagation through the local universe is mandatory.

When UHECRs propagate through the intergalactic medium (IGM) they lose energy in reactions with intergalactic background light and are deflected by extragalactic magnetic fields (EGMFs). These effects alter the cosmic ray spectrum and composition that is injected into the IGM by their sources. In order to get a better understanding about the origin and nature of UHECRs, it is of utmost importance to understand the imprint of the propagation and disentangle it from the UHECR properties at their sources. This can be achieved by comparing the measurements on UHECRs with model predictions. For this purpose extensive simulations of the propagation of UHE nuclei and their secondaries within given scenarios are required. CRPropa $2.0^{1}$ [9], a publically available Monte Carlo code, allows for such simulations.

With CRPropa 2.0 the extragalactic propagation of UHE nuclei in realistic one- (1D) and three-dimensional (3D) scenarios can be simulated. In these simulations all relevant particle interactions as well as magnetic deflections can be taken into account. Furthermore the propagation of all secondaries produced in the various reactions, including $\gamma$-rays and neutrinos, can be added. In this paper an overview is given of the different features of CRPropa, including as well two example simulations.

Throughout this paper natural units $\hbar=c=1$ are used.

\section{Source properties and distribution}

At the moment little is known about the sources of UHECRs. Possible candidates are for example AGNs and gamma ray bursts (GRBs). In CRPropa source candidates can be simulated by specifying their distribution, injection spectra and the UHECR composition at the source. Both in $1 \mathrm{D}$ and $3 \mathrm{D}$ a continuous source distribution, following a certain spacial density, or discrete sources, randomly drawn from a spacial density or specified by hand, can be implemented. The injection spectra can be specified either as a power law with a certain maximum energy or rigidity, or monochromatically. The injected composition can be any combination of nuclei up to mass number $A=56$.

\footnotetext{
${ }^{1}$ Available at https://crpropa.desy.de
} 


\section{Propagation}

When propagating through the IGM, UHECRs can interact with ambient photon backgrounds such as the cosmic microwave background (CMB) and the cosmic infrared background (IRB). They can undergo pair production, pion production and, in the case of nuclei, photodisintegration. When heavy nuclei react in one of these ways unstable nuclei can be produced, so the possible decay of these nuclei has to be taken into account as well. All these processes are included in CRPropa and will be discussed in more detail in the next sections. In these processes secondary photons, $e^{+} e^{-}$pairs and neutrinos can be produced. CRPropa allows as well for the simulation of the production and propagation of these secondaries. A module [10] is included that solves the one-dimensional transport equations for electromagnetic cascades that are initiated by electrons, positrons or photons taking into account single, double and triple pair production as well as up-scattering of low energy background photons by inverse Compton scattering. Furthermore it is possible within CRPropa to simulate the deflection of UHECRs and their secondaries within EGMFs. In the 1D mode the effects of magnetic fields are restricted to energy losses of $e^{+} e^{-}$pairs due to synchrotron radiation within electromagnetic cascades. However it is possible, in the 1D mode only, to specify the cosmological and source evolution as well as the redshift scaling of the background light intensity.

\subsection{Pair Production}

One of the relevant interactions for the propagation of UHECRs is the creation of $e^{+} e^{-}$pairs on the CMB and IRB. This is the most important reaction for the creation of secondary photons in the $\mathrm{TeV}$ range. The mean free path in this case is relatively short, however the energy loss in each individual interaction is relatively small (about $2 m_{e} / m_{p} \approx 0.1 \%$ per interaction, with $m_{e}$ the electron mass and $m_{p}$ the proton mass). Thus pair production can be treated to good approximation as a continuous energy loss, which is what is done within CRPropa. For details of the implementation see Ref. [9].

\subsection{Pion Production}

Furthermore secondary mesons can be produced by UHECRs in interactions with low energy photon backgrounds. The production of pions in reactions of UHE protons with the CMB, which leads to the GZK cut-off [7, 8], is an example of this type of interaction. For photopion production the energy loss per interaction is about $m_{\pi} / m_{p} \approx 15 \%$, with $m_{\pi}$ the pion mass. Thus this process is treated in a stochastic way, taking into account the mean free paths of all interaction channels. The mean free path for nucleons is modeled by the SOPHIA package [11]. To obtain the mean free paths for nuclei an effective scaling law is employed with scalings of the cross sections with mass number $A$. For further details of this scaling law and the implementation of photopion production within CRPropa see Ref. [9].

\subsection{Photodisintegration}

When considering nuclei, and not just free protons or neutrons, the process of photodisintegration becomes important. For almost all nuclei there are several competing photodisintegration processes of different cross sections which need to be accounted for. In CRPropa the publically available TALYS software version 1.0 [12] is used to compute most photodisintegration cross sections. However as the nuclear models therein are only reliable for mass number $A \geq 12$, additional photodisintegration cross sections for light nuclei have been implemented. In total TALYS was applied for 287 isotopes up to iron and cross sections for 17 additional isotopes were modeled is different ways. TALYS considers the separation of neutrons, protons, deuterium, tritium, helium-3 and helium-4 nuclei and combinations thereof from the initial nucleus. In this way in total 78449 exclusive reaction channels are taken into account within CRPropa. For details of the implementation and of the way the 17 additional isotopes were modeled, see Ref. [9].

\subsection{Decay}

Both by photodisintegration and photopion production unstable particles can be produced, making nuclear decay a relevant process in UHECR propagation. The implemented decay channels in CRPropa are based on the NuDat2 database [13] and include 434 different unstable nuclei with mass number $A \leq 56$ and charge $Z \leq 26$. The decays are modeled as (a combination of) $\alpha$ and $\beta^{ \pm}$decays and the loss of single nucleons. For more details again see Ref. [9].

\section{Propagation Algorithm}

In CRPropa a propagation algorithm has been implemented that can handle the widely ranging reaction rates of UHE nuclei. In this algorithm has been assumed that all mean free paths $\lambda_{i}$, where $i$ runs over all $N$ possible interaction and decay channels, are approximately constant during a time step. As $\lambda=\lambda(E)$ is in general a function of the UHECR energy $E$, the numerical step size has to be small enough to ensure that no significant energy loss occurs. The algorithm works as follows:

1. The inverse total mean free path $\lambda_{\mathrm{tot}}^{-1}=\left(\sum_{i=1}^{N} \lambda_{i}\right)^{-1}$ is calculated. From $\lambda_{\text {tot }}$ a distance $\Delta x_{1}$ to the next reaction is obtained in the following way

$$
\Delta x_{1}=-\lambda_{\text {tot }} \ln (1-r),
$$

using a uniformly distributed random number $0 \leq$ $r \leq 1$.

2. The fractional energy loss due to pair production is limited by imposing a maximum step size $\Delta x_{2}$ given by

$$
\int_{x}^{x+\Delta x_{2}} d x \frac{d E_{A, Z}^{e^{+}} e^{-}}{d x}(E)<\delta E,
$$

where $\delta$ is the maximal allowed fractional energy loss. 
3. The distance over which the particle will be propagated is calculated by

$$
\Delta x=\min \left(\Delta x_{1}, \Delta x_{2}, \Delta x_{3}\right),
$$

where $\Delta x_{3}$ is an upper limit on the propagation step that can be provided by the user, with typical values of $\Delta x_{3} \sim 0.1-50 \mathrm{Mpc}$. This increases the accuracy of the calculation of pair production energy losses, the accuracy of the $\gamma$-ray spectra coming from pair production and the accuracy in the determination of the deflections in magnetic fields.

4. If the particle ends up in a observer area after the propagation step $\Delta x$ the particle is considered detected and no further actions are performed.

5. If the UHECR does not reach an observer and $\Delta x=$ $\Delta x_{1}$, the particle is propagated over the path length $\Delta x_{1}$, where it performs an interaction. The specific interaction undergone by the UHECR is obtained by finding the smallest index $i$ for which

$$
\sum_{a=1}^{i} \frac{\lambda_{\mathrm{tot}}}{\lambda_{a}}>w,
$$

for a uniformly distributed random number $0 \leq w \leq$ 1. If $\Delta x=\Delta x_{2}$ or $\Delta x_{3}$ and does not reach an observer the UHECR is propagated over that specific path length but no interaction occurs.

6. The continuous energy losses are applied and the new particles produced in the interaction are added to the list of particles to be propagated. This algorithm is restarted again from the new position.

\section{Observer}

The observer can be specified in three different ways within CRPropa. In the $1 \mathrm{D}$ mode a particle is detected when its position is closer to the origin than the minimal step size, which can be specified in the input file. In 3D two different options are possible for the type of observer. One possibility is that the particle is detected when its position reaches a certain distance from the source, in which case only one discrete source should be specified. Observer spheres of different radii can be implemented at the same time. In the other possible case observers are defined by small spheres at positions and of radii of the users choice. A particle is recorded each time it enters one of these spheres.

\section{Examples}

In this section two examples are given, one for a $1 \mathrm{D}$ simulation and one for a 3D simulation. In the 1D case cosmological and source evolution as a function of the redshift is implemented. Furthermore the simulation of secondary neutrinos and $\gamma$-rays are included in this scenario. In the $3 \mathrm{D}$ example the effects of a source density and
EGMF following the large scale structure (LSS) baryon density are shown. The LSS-EGMFs used in this scenario were obtained from the cosmological simulations given in Ref. [14].

\subsection{D Example}

In Fig. 1 the results of two simulations are shown which differ only in the composition injected at the source, namely a galactic mixed composition (obtained from Ref. [21], similar to the approach of Ref. [22]) and a pure iron injection. In this way it is possible to investigate the effects of the poorly known initial composition. The additional parameters in these simulations are as follows: the UHECRs are injected with an $E^{-2.2}$ spectrum from a minimal energy of $1 \mathrm{EeV}$ up to a rigidity of $R=E / Z=384.6 \mathrm{EV}$. They originate from a continuous source distribution with comoving injection rate scaling as $(1+z)^{4}$ up to $z_{\max }=2$. The cosmological evolution is characterized by a concordance $\Lambda \mathrm{CDM}$ universe with a cosmological constant $\left(\Omega_{m}=0.3, \Omega_{\lambda}=0.7\right)$ using a Hubble constant of $H_{0}=72 \mathrm{~km} \mathrm{~s}^{-1} \mathrm{Mpc}^{-1}$. The simulated UHECR flux is normalized to the spectrum observed by the Pierre Auger Observatory $[15,16]$ at an energy of $10 \mathrm{EeV}$ and the secondary $\gamma$-ray and neutrino fluxes are normalized accordingly. The neutrino limits and spectra are all for single flavor neutrinos, assuming a ratio of $1: 1: 1$. The reduction of the flux of secondary neutrinos and $\gamma$-rays for pure iron injection, shown in Fig. 1(b), relative to an injected mixed galactic composition, Fig. 1(a), is due to photodisintegration dominating with respect to photopion production for the heavier composition. Both scenarios are however compatible with the single-flavor neutrino limits [17-19] and measured diffuse $\gamma$-ray flux [20].

\subsection{D Example}

In the 3D simulations of Fig. 2 the effect of the presence of a LSS-EGMF on the UHECR spectrum and composition is investigated. In this scenario a $(75 \mathrm{Mpc})^{3}$ simulation box with periodic boundary conditions filled with the continuous source distribution following the LSS baryon density and the corresponding LSS-EGMF in the scenario of Ref. [14] has been implemented. The further parameters of these simulations are as follows: the UHECRs are injected with an $E^{-1}$ spectrum, which is reweighted afterwards to a power law of $d N / d E \propto E^{-2.2}$ (in order to achieve sufficient statistics at high energies), from a minimal energy of $1 \mathrm{EeV}$ up to a rigidity of $R=E / Z=384.6 \mathrm{EV}$. A pure iron, pure proton and mixed galactic composition are injected. The detection occurs on a sphere centered around the observer with a radius of $\simeq 1 \mathrm{Mpc}$. The observer is placed in a magnetic environment that is similar to what is found in the vicinity of our Galaxy. The maximum propagation time that the UHECRs have been allowed to propagate corresponds to $3000 \mathrm{Mpc}$.

Fig. 2(a) shows that the shape of the spectrum changes for heavy versus light injected compositions. Furthermore, the simulated spectra are not strongly affected by the presence of the LSS-EGMF or LSS source density. In contrast, 


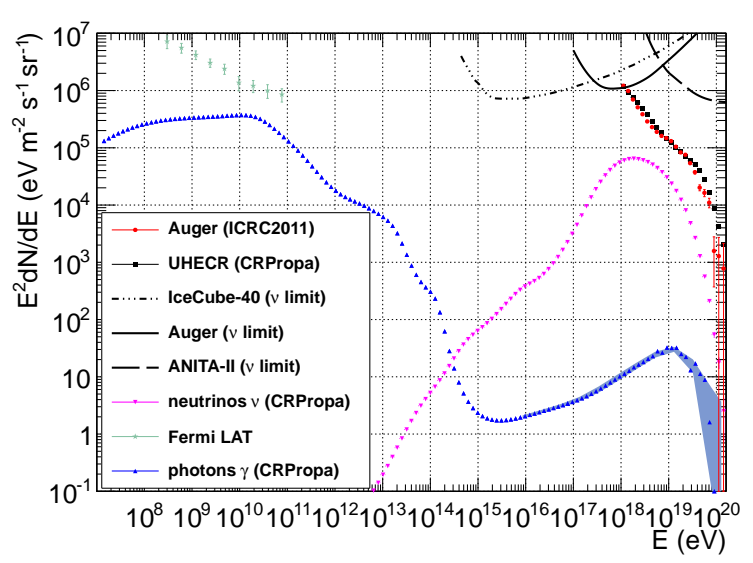

(a) injected galactic composition

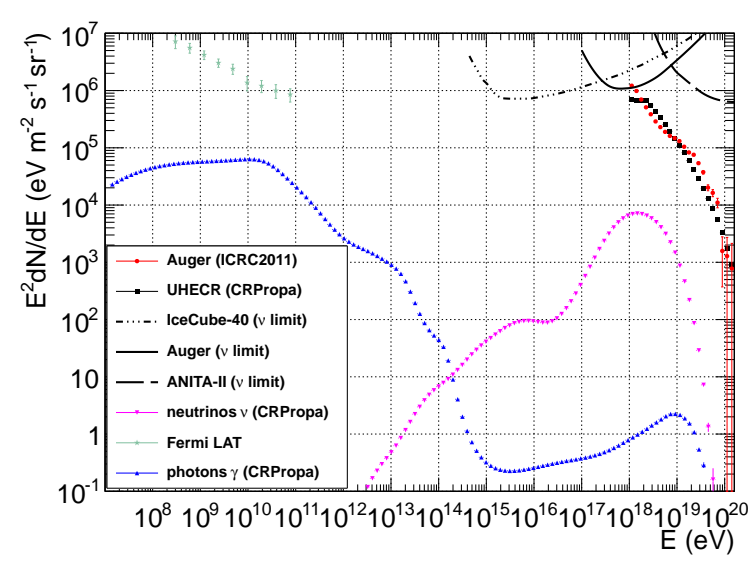

(b) pure iron injection

Figure 1. Results obtained from Ref. [9] of a 1D simulation with CRPropa taking into account cosmological expansion and a comoving source evolution scaling as $(1+z)^{4}$ up to $z_{\max }=2$. The simulated UHECR flux (black rectangles) has been normalized to the Pierre Auger spectrum (red dots) $[15,16]$. The spectra of secondary $\gamma$-rays (blue triangles) and neutrinos (magenta triangles) have been normalized accordingly. The neutrino flux shown is the single-flavor flux, assuming a ratio of 1:1:1. This flux can be compared with the single-flavor neutrino limits (black lines) [17-19]. Green stars show the isotropic $\gamma$-ray flux measured by Fermi-LAT [20]. In the left panel a galactic mixed composition has been injected at the source while in the right panel a pure iron composition has been injected.

the propagated composition of the pure iron injection case is affected by deflections, as shown in Fig. 2(b). This is due to deflections increasing the propagation path length, thereby enhancing interactions and reducing the average mass number $\langle A\rangle$ at detection. Finally, Fig. 2(c) illustrates that the LSS-EGMF has a strong effect on the horizon of the observer (for energies $E \gtrsim 1 \mathrm{EeV}$ ), especially for the heavier injected compositions. This is due to increased deflections and thus more interactions when heavier nuclei are injected.

\section{Conclusions}

In Ref. [9] a new version of the public UHECR propagation code CRPropa, a numerical tool to study the effect of extragalactic propagation on the spectrum, chemical composition and distribution of arrival directions of UHECRs on Earth, has been introduced. It can handle all relevant interactions for UHECR propagation, including the production and propagation of secondary neutrinos and $\gamma$-rays. Highly customizable realizations of source distributions and magnetic fields can be implemented. With these possibilities it can be used to compute the main observable quantities related to UHECR propagation, namely particle spectra, mass composition and arrival direction on Earth, with the accuracy required by present data.

\section{Acknowledgements}

This work was supported by the Deutsche Forschungsgemeinschaft through the collaborative research centre SFB 676, by BMBF under grants 05A11GU1 and 05A11PX1, and by the "Helmholtz Alliance for Astroparticle Phyics (HAP)" funded by the Initiative and Networking Fund of the Helmholtz Association. GS acknowledges support from the State of Hamburg, through the Collaborative Research program "Connecting Particles with the Cosmos". LM acknowledges support from the Alexander von Humboldt foundation.

\section{References}

[1] J. Abraham et al. (Pierre Auger Collaboration), Science 318, 938 (2007), 0711. 2256

[2] J. Abraham et al. (Pierre Auger Collaboration), Phys.Rev.Lett. 104, 091101 (2010), 1002.0699

[3] R. Abbasi et al. (HiRes Collaboration), Phys.Rev.Lett. 104, 161101 (2010), 0910. 4184

[4] J.N. Matthews (Telescope Array Collaboration), Nucl.Phys.Proc.Suppl. 212-213, 79 (2011)

[5] J. Abraham et al. (Pierre Auger Collaboration), Phys.Rev.Lett. 101, 061101 (2008), 0806. 4302

[6] R. Abbasi et al. (HiRes Collaboration), Phys.Rev.Lett. 100, $101101 \quad$ (2008), astro-ph/0703099

[7] K. Greisen, Phys.Rev.Lett. 16, 748 (1966)

[8] G. Zatsepin, V. Kuzmin, JETP Lett. 4, 78 (1966), engl. translation

[9] K.H. Kampert, J. Kulbartz, L. Maccione, N. Nierstenhoefer, P. Schiffer, G. Sigl, A.R. van Vliet (2012), to appear in Astropart. Phys., 1206. 3132

[10] S. Lee, Phys.Rev. D58, 043004 (1998), astro-ph/9604098

[11] A. Mucke, R. Engel, J. Rachen, R. Protheroe, T. Stanev, Comput.Phys.Commun. 124, 290 (2000), astro-ph/9903478

[12] A. Koning et al., TALYS-1.0, in Proceedings of the International Conference on Nuclear Data for Science and Technology (EDP Sciences, 2008), pp. 211-214 


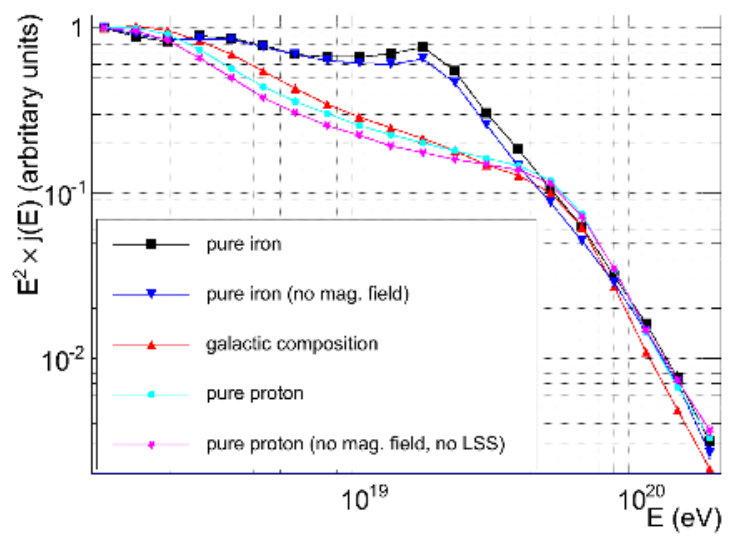

(a) energy spectra

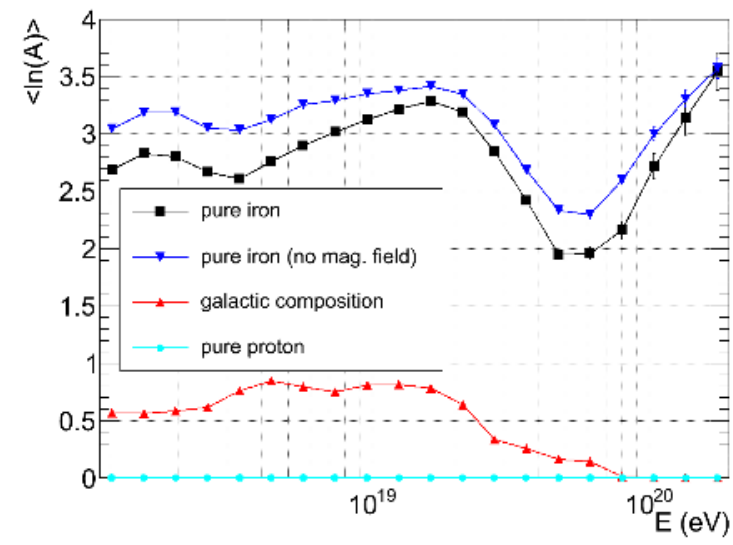

(b) mass spectra

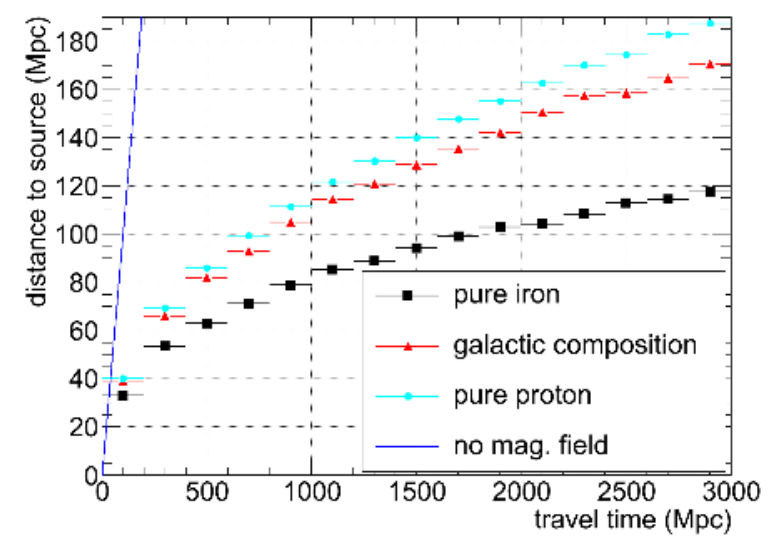

(c) source distance vs travel time

Figure 2. Example simulations of a continuous source distribution which follows the LSS density in the scenario of Ref. [14], obtained from Ref. [9]. Magnetic deflections of the UHECRs in the corresponding LSS-EGMF are taken into account in this 3D simulation. A pure iron composition (black rectangles), a galactic composition (red triangles) and a pure proton composition (light blue dots) have been injected at the sources.

(a), (b) UHECR flux and average mass number $\langle A\rangle$ as function of the energy at observation. Apart from the three cases for injected compositions mentioned before, which are shown including deflections in the LSS-EGMF, a pure iron simulation without deflection is shown for comparison (dark blue triangles). In the case of the UHECR flux a simulation with pure proton injection without deflections and with a flat continuous source distribution (not following the LSS density) has been added for comparison as well (magenta stars). The flux is normalized to unity in the first bin to allow for a better comparison of the spectral shape.

(c) Distance of the UHECRs from their source as function of the propagation time for all cosmic rays above $1 \mathrm{EeV}$, including a scenario without deflections (dark blue line).

[13] National Nuclear Data Center, NuDat 2 database, See http://www.nndc.bnl.gov/nudat2/ (2011)

[14] G. Sigl, F. Miniati, T. Ensslin, Nucl.Phys.Proc.Suppl. 136, 224 (2004), astro-ph/0409098

[15] J. Abraham et al. (Pierre Auger Collaboration), Phys.Lett. B685, 239 (2010), 1002 . 1975

[16] P. Abreu et al. (Pierre Auger Collaboration) (2011), 1107.4809

[17] R. Abbasi et al. (IceCube Collaboration), Phys.Rev. D83, 092003 (2011), 1103. 4250
[18] P. Abreu et al. (Pierre Auger Collaboration), Phys.Rev. D84, 122005 (2011), 1202 . 1493

[19] P. Gorham et al. (ANITA Collaboration), Phys.Rev. D85, 049901 (2012), 1011.5004

[20] A. Abdo et al. (Fermi-LAT collaboration), Phys.Rev.Lett. 104, 101101 (2010), 1002.3603

[21] M.A. Duvernois, M.R. Thayer, Astroph. Journal 465, 982 (1996)

[22] D. Allard, E. Parizot, E. Khan, S. Goriely, A. Olinto, Astron.Astrophys. 443, L29 (2005), astro-ph/0505566 